\title{
Factors Associated With Failed Induction of Labour in Patients Undergoing Induction with Titrated Oral Misoprostol at Harare Maternity Hospital
}

\section{B MATEVEKE (MBChB, MMED OBGYN) ${ }^{\text {a }}$ TCHIPATO (MBChB, FRCOG) ${ }^{\text {B }}$ B T GUZHA (MBChB, MRCOG) $^{a}$ L MAHACHI (BDS, MSc Biostatis) ${ }^{\mathrm{b}}$}

aDepartment of obstetrics and gynecology, University of Zimbabwe College of Health sciences, P O Box A178, Avondale

${ }^{\text {b}}$ Department of dentistry, University of Zimbabwe College of Health sciences, P O Box A178, Avondale

bmateveke@gmail.com

Study Synopsis: Maternal body mass index, total dose of misoprostol and the Bishop score were found to be significantly associated with failed labour induction.

\section{Abstract:}

Objective: To assess factors associated with failed induction in patients induced with oral misoprostol at 37 completed weeks of gestation or more.

Methods: A prospective cohort study was conducted on pregnant women with singleton fetuses in cephalic presentation undergoing induction of labour with titrated oral misoprostol at a tertiary hospital in Zimbabwe. The sample size was 171 . Participants were given between $15 \mathrm{ml}$ to $30 \mathrm{ml}$ of a $1 \mathrm{mcg} / \mathrm{ml}$ solution of oral misoprostol hourly and this was discontinued following the onset of labour pains. The main outcome measure was vaginal delivery within 24 hours of completing one cycle of titrated oral misoprostol.

Results: Only maternal body mass index (hazard ratio: 0.952; p value $=0.026$ ), the total dose of misoprostol (hazard ratio of 0.977 ; $p$ value $<0.001$ ), and the Bishop score (chi square 17.72; $p$ value $=<0.001$ ) were significantly associated with the outcome of induction. The rate of failed labour induction was $24.9 \%$.

Conclusion: Maternal body mass index, the total dose of misoprostol and the Bishop score significantly affected outcome of induction. The rate of failed induction with oral misoprostol was high but this was significantly reduced by repeating induction of labour when indicated.

Keywords: Failed induction of labour; oral misoprostol.

\section{INTRODUCTION}

Induction of labour is the artificial stimulation of uterine contractions before the spontaneous onset of labour, in order to achieve vaginal birth, when the risks of continuation of the pregnancy outweigh the benefits. . The WHO global survey on maternal and perinatal health showed an overall rate of induction of labour of $9.6 \%$ with the highest rates being found in Asian and Latin American countries (Sri Lanka 35.5\%) and the lowest rates in African countries (Niger 1.4\%) . 
Factors Associated With Failed Induction of Labour in Patients Undergoing Induction with Titrated Oral Misoprostol at Harare Maternity Hospital

Induction of labour can be achieved through various non pharmacological (mechanical) and pharmacological methods. Misoprostol is now being widely used off label for cervical ripening and induction off labour due to its low cost, availability and uterotonic activity. Many different routes of administration (rectal, vaginal, sublingual and oral) have been evaluated. The oral route has been the subject of many trials and different regimes have been employed.

Due to the wide use of oral misoprostol for labour induction, we assessed factors that were associated with failed labour induction in order to positively shape success rates and reduce the caesarian section rate as well as morbidity associated with caesarian delivery and elective repeat caesarian section.

\section{Materials AND Methods}

A prospective cohort study was undertaken from the $1^{\text {st }}$ of March 2013 to the $31^{\text {st }}$ of June 2013. A purposive sample of 171 pregnant women at 37 completed weeks of gestation or greater with singleton fetuses in cephalic presentation and with no previous history of caesarian section was recruited. Study participants were followed up from time of induction till discharge from hospital. Two of the participants did not meet the inclusion criteria leaving the final sample at 169 participants. Women with multifetal gestation, non cephalic fetal presentations or any contraindication to vaginal delivery were excluded.

Once selected for the study, each subject completed the process of informed consent and signed the consent form. Relevant maternal history and demographic data were collected and filled onto a data sheet. A thorough physical examination (including body mass index calculation, obstetric examination and bishop score assessment) was performed and the induction procedure started by the midwives on duty that day. A $200 \mathrm{mcg}$ tablet of misoprostol was dissolved in $200 \mathrm{ml}$ of tape water to make a $1 \mathrm{mcg} / \mathrm{ml}$ solution. Aliquots between 15 to $30 \mathrm{mls}$ were given orally every hour according to the Harare hospital maternity induction regimen in use at the time (see figure 1). This was stopped once labour pains had started and the patients were sent to labour ward.

Table1. Harare hospital maternity oral misoprostol regime (during the study period).

\begin{tabular}{|l|l|l|}
\hline PARITY & LOADING DOSE & HOURLY DOSE \\
\hline Para 0 & $30 \mathrm{ml}^{*}$ & $20 \mathrm{ml}^{*}$ \\
\hline Para 1 and 2 & $20 \mathrm{ml}^{*}$ & $15 \mathrm{ml}^{*}$ \\
\hline Para 4( with caution) & $15 \mathrm{ml}^{*}$ & $15 \mathrm{ml}^{*}$ \\
\hline
\end{tabular}

200microgrammes misoprostol dissolved in $200 \mathrm{ml}$ saline to make a 1microgrammes/ml solution

Intrapartum management of the patients was done by the obstetric team on duty that day and patients were sent to the postnatal ward once delivered. There the principal investigator would follow them up and consolidate information on delivery outcomes and the outcome for the neonate. Caesarean section was done for any obstetric emergencies during labour.

Those who failed to enter into labour or did not deliver within 24hours of completing the induction process were followed up every day to evaluate their subsequent management, until delivery (caesarean section or otherwise) and routine postnatal follow up was done as before.

The maternity booklet, misoprostol induction chart, and delivery registers were used to assist with consolidation of the study information.

An analysis was then carried out to assess study factors associated with failed induction using IBM SPSS Statistics version 21 with assistance from a statistician. 
Factors Associated With Failed Induction of Labour in Patients Undergoing Induction with Titrated Oral Misoprostol at Harare Maternity Hospital

Study participants were furnished with information leaflets informing them about the study in English and Shona languages including risks and benefits to them. Written Informed consent was obtained and those who did not wish to take part were excluded and were not penalized. Issues of confidentiality were discussed with the participants and the information was provided in written format on each consent form. Ethical approval was sought from Harare hospital institutional ethics board, the Joint Research and Ethics Committee (JREC ) and the Medical Research Council of Zimbabwe (MRCZ).

\section{RESULTS}

The median age was 24 years with age range from 16years to 42years. Ninety six percent of participants were married, with $85 \%$ having reached ordinary level education and majority of the participants were not employed (69.6\%). Forty one percent of the participants were nulliparous and only $6.5 \%$ had 3 or more children. The commonest indications for induction were hypertension in pregnancy $(38.1 \%)$, followed by prolonged pregnancy (33.9\%) and prelabour rupture of membranes at term (16.1\%). Only one participant had induction done for intrauterine fetal demise at term.

Ninety percent of the subjects had a Bishop score $<5$ with only $10.1 \%$ having a score of 6 or greater and 81,7\% had unruptured membranes at presentation. The mean birth weight at delivery was 3078g. The median delivery time was 16.7 hours (95\% CI: 14.67 hours; 17.67 hours). The median dose of misoprostol used was 170 microgrammes ( $95 \%$ CI: 165microgrammes; 180 microgrammes), with a range from 20 microgrammes to 215microgrammes. Twenty five percent of the participants had a failed induction by definition, this however decreased to $19 \%$ after a repeat induction with oral misoprostol. some patients had repeat induction with "oral" misoprostol and if these were included in the cohort of participants with failed Labour induction, the failure rates of induction would reduce to further to $19 \%$ after 2 inductions.

Multivariate analysis using Cox-regression model of all materno-fetal indicators showed only maternal body mass index (BMI) and the total dose of misoprostol used were significantly associated with outcome of induction using this model.

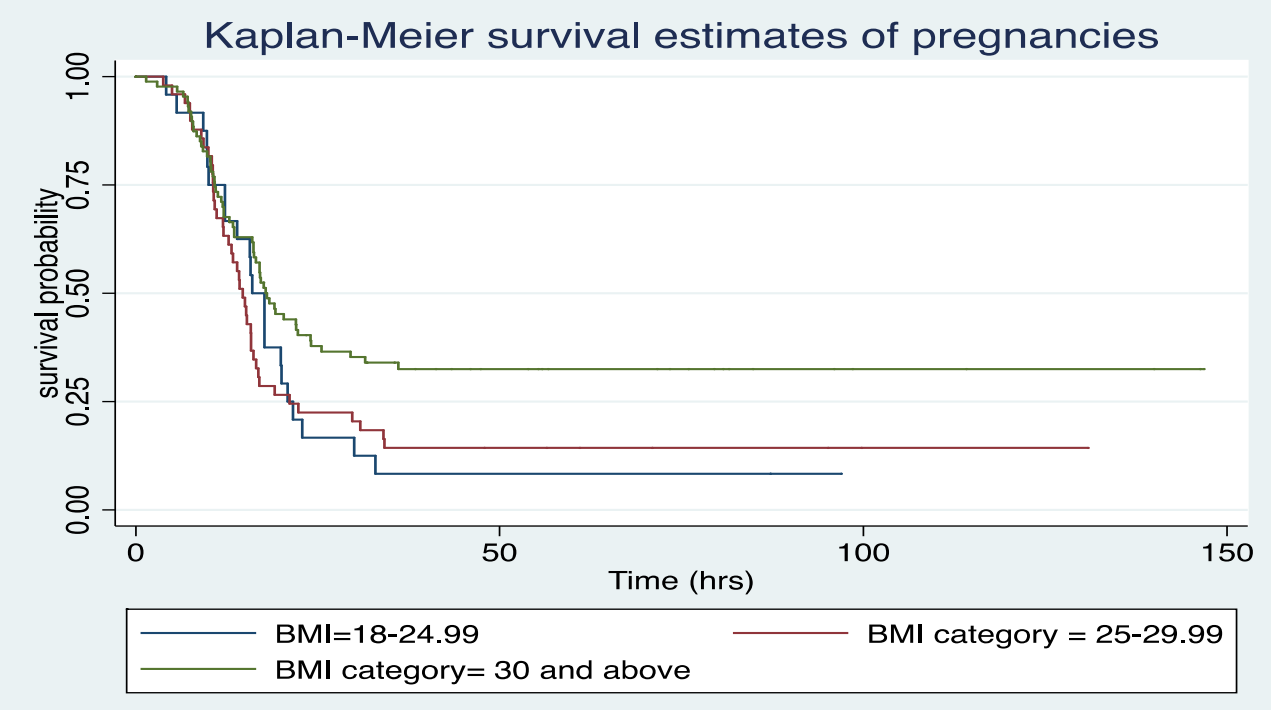

Fig1. Kaplan-Meier survival estimates for pregnancies by BMI category

The table below shows the Cox-regression for the association with outcome of induction, with P-values, for all the maternal and fetal factors evaluated in the study. 
Factors Associated With Failed Induction of Labour in Patients Undergoing Induction with Titrated Oral Misoprostol at Harare Maternity Hospital

Table2. Statistical significance of association between materno-fetal factors and outcome of induction

\begin{tabular}{|l|l|}
\hline Variable & P value \\
\hline BMI & 0.026 \\
\hline Total dose of misoprostol & $<0.001$ \\
\hline Age & 0.576 \\
\hline Parity & 0.565 \\
\hline Indication for induction & 0.520 \\
\hline State of fetal membranes & 0.168 \\
\hline Birth weight & 0.390 \\
\hline Bishop score & 0.218 \\
\hline
\end{tabular}

Univariate analysis (unadjusted estimates) using the log rank test for equality of survival functions was performed for BMI, Bishop Score and parity and noted that BMI category was indeed associated with outcome of induction ( chi square 7.3; P value $=0.026$ ) as well as bishop score category (chi square 17.72; P value<0.001). Parity was not associated with outcome of induction (chi square 2.04; P value $=0.565$ ).

\section{DiscusSiON}

The study found that induction failed in $24.9 \%$ of women induced with titrated oral misoprostol, meaning that about 1 in 4 women induced with our Harare hospital regime end up with a failed induction. A local study comparing effectiveness of oral misoprostol and vaginal misoprostol showed that $11 \%$ of women failed on titrated oral misoprostol regime ${ }^{2}$. Analysis of the cohort of women with failed labour induction showed that $19(45.2 \%)$ had a repeat induction with oral misoprostol and of these 10 succeeded in achieving vaginal delivery within 24 hours of completing the cycle. This reduces the failure rate to $19 \%$ after two inductions. This is further reduced to $16 \%$ if the women who had per vaginal misoprostol instead, are included together with those who had oral misoprostol.

Various studies on induction of labour in general (not limited to oral misoprostol) show varying failure rates up to as high as $25 \%$ percent in one study. The high failure rate in this study could be explained by the fact that the definition for failed induction was somewhat restrictive as anyone managing to deliver vaginally after the stipulated 24hours was considered as a failed induction (even if vaginal delivery occurred within 48hours).

Some patients despite getting into active labour within the stipulated 24hours ended up with caesarian sections for obstetric reasons during labour. They were also included in the cohort of failed inductions for the sole reason of failing to attain an important end point to induction of labour- vaginal delivery.

Whether the regime employed for induction is inferior to other standard regimes recommended by the WHO or others is not clear but it is actually more dose dense in terms dosing frequency compared to these other regimes $^{2,3,4,5}$. The study did not evaluate the adherence to the regime protocol in terms of dosing frequency, timing and when to stop administering the misoprostol as this may also have contributed to failures if not adhered to. Offering a repeat induction with the same regime or different regime, if indicated, does indeed help to reduce failures as shown in this study. Only 1 patient was induced for intrauterine fetal demise.

Most of the women who had a failed induction ended up with a repeat induction with oral misoprostol (45.2\%), with caesarian section for failed induction being done for $23.9 \%$. It was the second commonest intervention. 
Factors Associated With Failed Induction of Labour in Patients Undergoing Induction with Titrated Oral Misoprostol at Harare Maternity Hospital

Nineteen percent ended delivering without any intervention. Induction with vaginal misoprostol insertion was the least common intervention. There are no laid down standard criteria for options to follow once induction of labour has failed ${ }^{6}$. When to repeat the induction process or which intervention to offer patients generally varied between the obstetric teams managing the patients. Some patients were subjected to long periods of waiting before any intervention was given, going as long five days for some. When induction failed over a weekend most patients had to wait for a Monday when normal duties resume for them to be reviewed by the responsible teams as the on call team would mostly be occupied with work in labour ward.

This scenario calls for the need for continuous quality improvement of the management of failed labour induction through regulars case management audits in order to formulate a protocol to harmonise management of these patients and thus prevent potential adverse outcomes or unnecessary caesarian deliveries. If clinically indicated, this study has shown that indeed repeat induction does improve overall success rates. The essential guide to common obstetric and gynecological conditions in Zimbabwe does not say anything on management of failed labour induction ${ }^{4}$.

In this study, the only factors shown to be significantly associated with outcome of induction were the body mass index of the mother and the total dose of misoprostol. Univariate analysis showed bishop score category to be significantly associated with induction outcome. In contrast to most studies published on induction of labour and factors affecting success rates, we did not find any significant association between maternal age, parity and birth weight with the outcome of induction. The fact that the mean BMI for the study population was $31 \mathrm{~kg} / \mathrm{m}^{2}$ and the fact that a large proportion of women had a BMI $>30$ shows that obesity in pregnancy within our pregnant population is prevalent and deserves the necessary recognition in order to alleviate potential adverse outcomes associated with obesity in pregnancy including failed induction as demonstrated in this study.

There was significant correlation between the Bishop Score category and outcome of induction with oral misoprostol after univariate analysiss. This means that preinduction cervical assessment in the setting of induction with misoprostol is necessary even though misoprostol does induce both cervical ripening and uterine contractions. Compared to women with ruptured membranes, those who had induction of labour with unruptured membranes had higher induction failure rates (2\% vs $29 \%$ ). These results are in keeping with findings from other studies including a South African study by Hofmeyr and Alfirevic which showed a slower rate of response to induction in women with intact membranes and unfavorable cervix ${ }^{7}$.

In keeping with most studies that proved the safety and efficacy of oral misoprostol for labour induction, only one patient had a uterine rupture after which a hysterectomy was done, 7.1\% developed fetal distress and there was no documented evidence of uterine hypertonus in all the patients ${ }^{2,8,9,10}$

The one uterine rupture recorded occurred in a 26 year old para 1 gravida 2 at 38 weeks of gestation who had been induced for hypertension in pregnancy. She had a BMI of $44.9 \mathrm{~kg} / \mathrm{m}^{2}$. A total dose of 165 microgrammes of misoprostol was used for induction. Intrapartum, she was in active phase for close to $6 \mathrm{hrs}$ with no descent of the fetal head, a caesarian section was due to be done for failed progress and fetal bradycardia upon where a traumatic uterine rupture was noted. A total hysterectomy was done. She delivered a fresh still birth with weight of $3600 \mathrm{~g}$. There was no recorded of oxytocin use intrapartum.

In conclusion only maternal BMI, the total dose of misoprostol and bishop score were significantly associated with the outcome of induction. Routine assessment of body mass index and Bishop score should become standard practice before labour induction in order to help predict probability of a successful induction.

The rate of failed induction of labour using titrated oral misoprostol was $24.9 \%$ meaning that 1 in 4 women failed to achieve vaginal delivery when using the Harare hospital regime. A repeat induction if indicated 
Factors Associated With Failed Induction of Labour in Patients Undergoing Induction with Titrated Oral Misoprostol at Harare Maternity Hospital

reduced failure rates to as low as $16 \%$. The performance of this regime needs however to be compared to the current standard regimes adopted from the WHO that have since been adopted as guidelines in the labour ward protocols $^{1}$.

There are no standard treatment guidelines for the management of a failed labour induction; this can have a negative impact on the quality of care provided to mothers who fail at induction of labour. Setting up of such guidelines would go a long way in improving maternal health care.

\section{Conflict of interest}

All the authors have no conflict of interest to declare.

\section{REFERENCES}

[1] World Health Organisation. WHO Recommendations for induction of labour. WHO Press. Geneva. 2011 http://whqlibdoc.who.int/hq/2011/WHO_RHR_11.10_eng.pdf

[2] Zvandasara P, Saungweme G, et al. Induction of labour with titrated oral Misoprostol suspension. A comparative study with vaginal Misoprostol. Central African Journal of Medicine. 2008; 54: 43-9.

[3] 6th Essential Medicines List and Standard Treatment Guidelines for Zimbabwe. Ministry of Health and Child Welfare. WHO press. 2011

[4] Essential guide to management of common obstetric and gynecologic conditions in Zimbabwe. University of Zimbabwe, Department of Obstetrics and Gynaecology.2012; 8:30-32

[5] NICE Clinical Guidelines. Induction of labour. www.nice.org.uk/CG070.

[6] Vikram S. Talaulikar. Failed induction of labour strategies to improve success rates. Obstetrical and Gynecological survey. 2011. Vol 6, number 11:717-727

[7] Hofmeyr G.J, Alfirevic Z, et al. titrated oral Misoprostol solution for induction of labour: a multicentre randomized controlled trial. British journal of obstetrics and gynecology. 2001. Vol 108; 952-959

[8] Majoko F, Magwali T, Zwizwai M. uterine rupture associated with the use of Misoprostol for induction of labour. International Journal of Gynaecology and Obstetrics. 2002. 76:77-78.

[9] Majoko F, Nystrom L, etal. No benefit but increased harm from high dose Misoprostol for induction of labour : a randomized controlled trial of high vs low dose Misoprostol. Journal of Obstetrics and Gynaecology. 2002 Nov. 22(6):614-7

[10] Alfirevic Z, Weeks A. oral Misoprostol for induction of labour. Cochrane database of systemic reviews. 2006.

Citation: B MATEVEKE (MBChB, MMED OBGYN), TCHIPATO (MBChB, FRCOG), B T GUZHA (MBChB, MRCOG), L MAHACHI (BDS, MSc Biostatis). "Factors Associated With Failed Induction of Labour in Patients Undergoing Induction with Titrated Oral Misoprostol at Harare Maternity Hospital". American Research Journal of Gynaecology, 1(1): 43-48.

Copyright (C) B MATEVEKE (MBChB, MMED OBGYN), TCHIPATO (MBChB, FRCOG), B T GUZHA (MBChB, MRCOG), L MAHACHI (BDS, MSc Biostatis). This is an open access article distributed under the Creative Commons Attribution License, which permits unrestricted use, distribution, and reproduction in any medium, provided the original work is properly cited. 